\title{
Changements de comportement en matière de santé autodéclarés chez les adultes : une analyse de l'Enquête sur la santé dans les collectivités canadiennes, cycle 4.1
}

\author{
C. Haberman, M. Sc., RD (1); P. Brauer, Ph. D., RD (1); J. J. Dwyer, Ph. D. (1); A. M. Edwards, Ph. D. (2)
}

Cet article a fait l'objet d'une évaluation par les pairs.

\section{Résumé}

Introduction : Connaître l'expérience des Canadiens ayant trait aux changements de comportement en matière de santé (CCS), que ce soit en général ou celle des personnes à risque en raison de leur indice de masse corporelle (IMC), serait utile à l'élaboration de programmes de promotion de la santé et de prévention des maladies. Nous avons ainsi examiné, dans le cadre d'une analyse secondaire du cycle 4.1 de l'Enquête sur la santé dans les collectivités canadiennes, une sélection de CCS autodéclarés au cours des douze derniers mois, par catégorie d'IMC : faire plus d'exercice ou de sport, perdre du poids et améliorer ses habitudes alimentaires. Nous nous sommes aussi penchés sur les obstacles aux CCS.

Méthodologie : Nous avons analysé les données provenant des répondants de 18 ans et plus à l'aide d'une méthode de régression logistique progressive ascendante. Les IMC autodéclarés ont été corrigés à l'aide de la méthode de Connor Gorber et ses collaborateurs (2008).

Résultats : Notre échantillon final était de 111449 répondants. Sur l'ensemble des répondants, $58 \%$ avait fait un CCS, l'augmentation de la pratique sportive ou d'exercice étant le plus important (29\% des répondants), suivi par l'amélioration des habitudes alimentaires (10\%) et la perte de poids (7\%). La moitié (51\%) des répondants avaient rencontré des obstacles, le manque de volonté étant l'obstacle le plus souvent mentionné, suivi par l'emploi du temps professionnel et les responsabilités familiales. Les répondants obèses ont fait état de CCS plus souvent que les répondants dont le poids était normal ( $60 \%$ contre $55 \%$ ), mais la fréquence de l'augmentation de la pratique sportive ou d'exercice et de l'amélioration des habitudes alimentaires était similaire selon toutes les catégories d'IMC. Les modèles de régression ne représentaient que de $6 \%$ à $10 \%$ de la variance totale.

Conclusion : Le fait que la majorité des répondants ait tenté d'apporter au moins un CCS est de bon augure pour une évolution positive de la santé de la population. D’autres travaux sont nécessaires pour mieux caractériser la population et pour améliorer les indicateurs qui lui sont associés, et ainsi évaluer l'incidence des efforts de promotion de la santé et de prévention des maladies. Nos résultats fournissent des données de référence pouvant servir de base à ces travaux.

Mots-clés : comportement en matière de santé, obésité, perte de poids, régime

alimentaire, activité physique, caractéristiques de la population

\section{Introduction}

Grâce à l'élaboration de l'Enquête sur la santé dans les collectivités canadiennes (ESCC) en 2000 et de l'Enquête canadienne sur les mesures de la santé en 2009, les planificateurs des services de santé ont amélioré leur capacité à évaluer correctement les répercussions de leurs efforts de promotion de la santé et de prévention des maladies sur la santé des Canadiens ${ }^{1}$. Parmi les nombreux enjeux de santé, l'obésité, dont les taux sont en hausse, est particulièrement digne d'intérêt en tant que facteur de risque intermédiaire de maladies chroniques courantes.

Actuellement, chez les adultes de 19 à 79 ans, la prévalence de l'excès de poids est de 34,2 \% et celle de l'obésité, de $26 \%{ }^{2}$. Le régime alimentaire et l'activité physique sont les principaux facteurs liés au mode de vie ayant un effet sur la prévalence de l'obésité. À ce jour, les enquêtes transversales sur la santé ont fourni des renseignements limités sur la fréquence de l'activité physique et de la consommation de fruits et légumes (en tant qu'outil de mesure d'un régime alimentaire sain). Un examen des tableaux sommaires 2009-2012 de l'ESCC indique que 56,2 \% des Canadiens de 12 ans et plus ont suffisamment d'activités de loisirs exigeant au moins une activité modérée $(1,5 \mathrm{kcal} / \mathrm{kg} / \mathrm{jour} \text { ou plus })^{2}$, et ce pourcentage demeure stable. Toutefois, la proportion de Canadiens qui consomment chaque jour cinq portions ou plus de fruits et légumes est passée de 45,6\% en 2009 à $40,6 \%$ en $2012^{3}$.

Rattachement des auteurs :

1. Département des relations familiales et de la nutrition appliquée, Université de Guelph, Guelph (Ontario), Canada

2. Centre de ressources, bibliothèque, Université de Guelph, Guelph (Ontario), Canada

Correspondance : Paula Brauer, 50, Stone Road E., Guelph (Ontario) N1G 2W1; tél. : 519-824-4120, poste 54831; téléc. : 519-766-0691; courriel : pbrauer@uoguelph.ca 
Les données sur la fréquence au sein de la population des changements de comportement en matière de santé (CCS) autodéclarés visant à améliorer le régime alimentaire, la pratique de l'activité physique et le poids - principaux obstacles aux changements - ainsi que les caractéristiques sociodémographiques qui leur sont associées, pourraient servir à élaborer de nouvelles mesures et fournir des données permettant d'évaluer la réussite à long terme des approches en santé publique $^{4,5}$. L'ESCC cycle 4.1 (2007) a permis d'examiner la fréquence des CCS et a fourni de l'information sur les variables individuelles et les variables de santé associées et sur les obstacles aux changements $^{6}$. Les objectifs de cette analyse secondaire du cycle 4.1 de l'ESCC étaient d'examiner la fréquence des CCS autodéclarés chez les adultes en général et par catégorie d'indice de masse corporelle (IMC), de relever les facteurs sociodémographiques associés aux CCS et d'étudier la fréquence des obstacles aux CCS par catégorie d'IMC.

\section{Méthodologie}

L'ESCC cycle 4.1 est une enquête nationale transversale dans laquelle les données sur l'état de santé, l'utilisation des soins de santé et les déterminants de la santé, dont les CCS, sont autodéclarées ${ }^{6}$. Les données ont été recueillies auprès de répondants de l'ensemble des provinces et des territoires, de sorte que les résultats sont représentatifs de $98 \%$ de la population canadienne. Une information plus détaillée sur l'enquête est disponible en ligne $^{6}$.

L'IMC a été calculé et réparti en classes conformément aux normes nationales de classification du poids ${ }^{7}$. Le biais lié à l'IMC autodéclaré, selon lequel les gens sousestiment leur poids et surestiment leur taille, est bien connu ${ }^{8}$. Comme le poids et la taille étaient autodéclarés dans cette étude, nous avons donc appliqué à l'IMC estimatif des adultes en excès de poids ou obèses les équations de correction déjà établies, mais pas à celui des adultes dont le poids était normal, en raison d'un biais de déclaration moins marqué dans ce groupe ${ }^{9}$.
L'analyse a porté uniquement sur les répondants de 18 ans et plus (en excluant les femmes enceintes) ayant répondu euxmême au questionnaire (et non par procuration) et dont l'IMC se situait entre $18,5 \mathrm{~kg} / \mathrm{m}^{2}$ et $55,0 \mathrm{~kg} / \mathrm{m}^{2}$ avant la correction apportée pour tenir compte du biais de déclaration. Les répondants qui se trouvaient à l'extérieur de cette fourchette d'IMC ont été exclus en raison des problèmes de santé spécifiques avec lesquels ils étaient aux prises en raison de leur poids.

L'autodéclaration de changement ou non de comportement en matière de santé au cours de l'année précédente était le principal résultat qui nous intéressait. Les participants devaient indiquer également le CCS le plus important qu'ils avaient fait au cours de l'année (voir la figure 1 pour l'ordre des questions), les choix de réponse étant d'avoir fait davantage d'exercice, de sport ou d'activité physique, d'avoir perdu du poids, d'avoir changé son alimentation ou amélioré ses habitudes alimentaires, d'avoir cessé de fumer ou réduit sa consommation de tabac, d'avoir consommé moins d'alcool, d'avoir réduit son niveau de stress, d'avoir reçu des traitements médicaux, d'avoir pris des vitamines ou d'avoir fait un autre changement. Les répondants ayant adopté plusieurs CCS devaient sélectionner le plus important.

On demandait aux répondants qui avaient indiqué ressentir le besoin de faire quelque chose pour améliorer leur santé d'énumérer les obstacles rencontrés aux CCS. Ils pouvaient choisir plusieurs obstacles parmi les suivants : manque de volonté ou d'autodiscipline, emploi du temps professionnel, responsabilités familiales, incapacité, problème de santé, trop de stress, coût élevé, conditions météorologiques, dépendance aux drogues ou à l'alcool, impossibilité dans l'environnement proche, problèmes de transports et autres obstacles non spécifiés. «Volonté » est le terme courant utilisé pour désigner la perception d'un contrôle de comportement, qui est un concept global comprenant deux sous-catégories, l'auto-efficacité et la contrôlabilité ${ }^{10,11}$.

Nous avons effectué des analyses statistiques descriptives (à l'aide de la version
$19.0 \mathrm{du}$ logiciel SPSS [IBM Corp., Armonk, N.Y., É.-U.]) pour définir les caractéristiques des répondants et déterminer la fréquence des CCS selon leur poids (poids normal, en excès de poids ou obésité). Lorsque le test du chi-carré indiquait une différence entre les proportions, nous avons comparé celles-ci au moyen d'un test $z$ (nous avons considéré que le théorème de la limite centrale s'appliquait) et nous les avons ajustées pour les comparaisons multiples à l'aide de la correction de Bonferroni. En outre, nous avons soumis les variables continues à une analyse de la variance avec comparaison a posteriori des moyennes à l'aide du T2 de Tamhane, sans présumer que les tailles ou les variances des échantillons étaient égales. Les données ont été pondérées au moyen des poids d'échantillonnage fournis $^{12}$. Nous avons ensuite effectué une analyse pour les sous-ensembles de répondants ayant fait ou non des CCS et ayant rencontré ou non des obstacles. Les personnes n'ayant pas ressenti le besoin de faire d'autre changement de CCS (figure 1) ont été exclues de l'analyse.

Nous avons utilisé un modèle de régression logistique progressive ascendante pour déterminer les variables associées aux CCS et les classer par ordre d'influence, tout en contrôlant pour l'âge, le sexe, le niveau de scolarié et le revenu personnel. Les premiers modèles de régression générés incluaient toutes les variables de l'ESCC qui avaient été associées à un changement de poids et à l'activité physique dans la revue de la littérature à laquelle nous avions préalablement procédé. Les variables étaient l'âge et le sexe, les caractéristiques sociodémographiques, le niveau de scolarité, le revenu, le statut d'immigration, l'état de santé général, la taille et le poids autodéclarés, les maladies chroniques, la médication, les changements faits pour améliorer la santé, la sécurité alimentaire, l'indice d'activité physique, la sédentarité, le soutien social et le stress au travail. Après vérification des chevauchements conceptuels, des enjeux temporels et de la multicolinéarité, nous avons retenu les meilleurs prédicteurs. Nous avons alors créé un modèle de régression pour les CCS dans leur ensemble et, en fonction des résultats obtenus, nous avons ensuite généré des modèles indivi- 
FIGURE 1

Diagramme de l'enchaînement des questions : module sur les changements de comportement en matière de santé

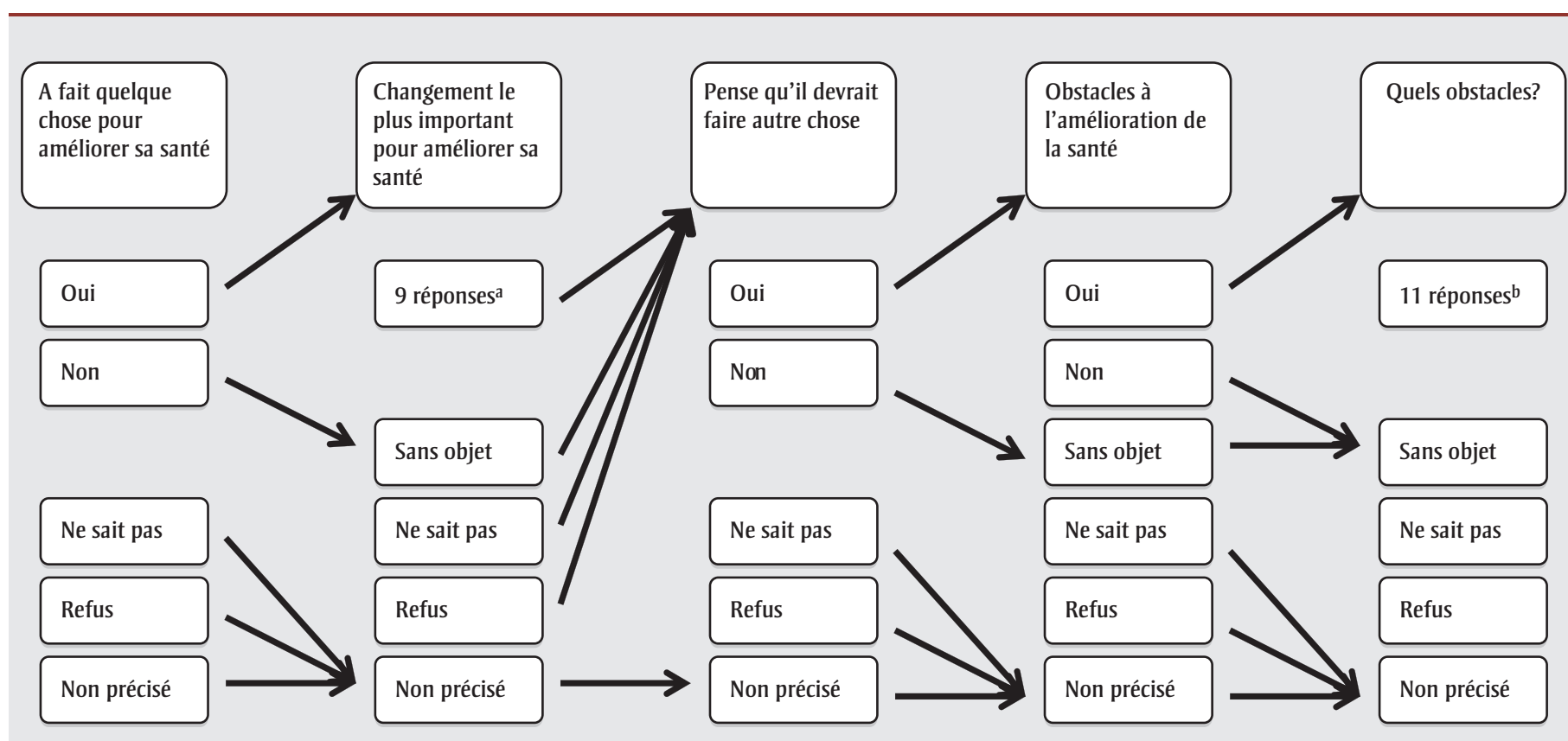

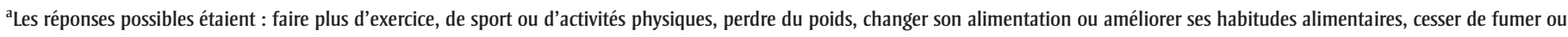
réduire sa consommation de tabac, consonsommer moins d'alcool, réduire son niveau de stress, recevoir des traitements médicaux, prendre des vitamines et autre.

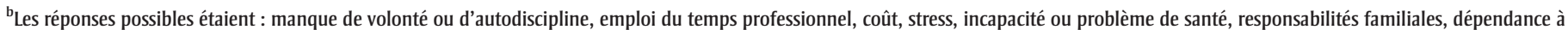
la drogue ou à l'alcool, mauvaise forme, environnement non favorable, problèmes de transports, conditions météorologiques et autre.

duels pour prédire chacun des CCS. Au départ, des modèles distincts ont été appliqués à chacune des catégories d'IMC mais, en raison des similitudes entre les modèles, toutes les catégories d'IMC ont finalement été regroupées et l'IMC a été inclus dans le modèle en tant que variable prédictive continue. Nous avons vérifié l'ajustement du modèle à l'aide du coefficient $R^{2}$ de Nagelkerke et du test de Hosmer-Lemeshow. Étant donné que les modèles ont révélé peu de variance, nous n'avons pas utilisé de poids d'échantillonage (bootstrap).

\section{Résultats}

Des 111449 répondants de l'échantillon final, $27 \%$ (n = 30 442) étaient obèses, $29 \%(\mathrm{n}=31831)$ étaient en excès de poids et $44 \%(n=49176)$ avaient un poids normal. Les variables sociodémographiques sélectionnées sont présentées dans le tableau 1.

Dans l'ensemble, 58 \% (n = 64 035) des répondants ont indiqué avoir pris des mesures pour améliorer leur santé (tableau 2). Le CCS le plus populaire auprès de l'ensemble des répondants était de faire davantage d'exercice, de sport ou d'activité physique (29\%, n = 31 936), suivi du changement d'alimentation ou de l'amélioration des habitudes alimentaires (10\%) et de la perte de poids (7\%). Les autres CCS (13\%) étaient d'avoir cessé de fumer ou réduit sa consommation de tabac, d'avoir consommé moins d'alcool, d'avoir réduit son niveau de stress, d'avoir reçu des traitements médicaux, d'avoir pris des vitamines et d'avoir effectué un autre changement non spécifié. Un pourcentage plus élevé de répondants obèses (60\%) que de répondants ayant un poids normal (55\%) a indiqué avoir tenté d'améliorer sa santé.

Les deux tiers (68 \%; $\mathrm{n}=75$ 717) des répondants estimaient qu'ils devaient en faire davantage pour améliorer leur santé. De ce nombre, 51 \% (n = 38 193) avaient rencontré un ou plusieurs obstacles à leurs CCS (voir le tableau 2), l'obstacle le plus souvent mentionné étant le manque de volonté ou d'autodiscipline (34\%), suivi de l'emploi du temps professionnel (28\%) et des responsabilités familiales (15\%). Moins de $10 \%$ des répondants ont affirmé avoir rencontré un ou plusieurs autres obstacles. Parmi les répondants ayant rencontré des obstacles à leurs CCS, ceux qui étaient obèses étaient beaucoup plus susceptibles que ceux dont le poids était normal de citer le manque de volonté, une incapacité ou un problème de santé ( $p<0,05$ pour l'ensemble). En revanche, les répondants dont le poids était normal étaient beaucoup plus susceptibles que les répondants obèses de citer le travail, les responsabilités familiales et les contraintes financières ( $p<0,05$ pour l'ensemble) (voir la figure 2).

Nous avons effectué une analyse par sousensembles pour mesurer les différences dans la fréquence des obstacles rencontrés chez les personnes qui ont indiqué avoir fait un CCS et chez celles qui n'en avaient pas fait. Sur le plan statistique, les responsabilités familiales représentaient un obstacle significativement plus élevé $(p<0,001)$ chez les personnes n'ayant pas fait de CCS (16\%) que chez celles qui en ayant fait (15\%), mais cet écart était 
TABLEAU 1

Caractéristiques sociodémographiques de la population de 18 ans et plus, par classe d'IMC, Canada, ESCC 2007

\begin{tabular}{|c|c|c|c|}
\hline Variables & $\begin{array}{l}\text { Obésité } \\
n=30442\end{array}$ & $\begin{array}{l}\text { Excès de poids } \\
\mathrm{n}=31831\end{array}$ & $\begin{array}{l}\text { Poids normal } \\
\mathrm{n}=49176\end{array}$ \\
\hline Sexe masculin ${ }^{\mathrm{a}}$ (en \%) & 53,5 & 61,0 & 43,3 \\
\hline Âge moyen (en années) (ÉT) ${ }^{\mathrm{b}}$ & $48,7(15,6)$ & $48,5(16,4)$ & $43,7(17,6)$ \\
\hline IMC moyen $\left(e n ~ k g / m^{2}\right)(E ́ T)^{\mathrm{a}}$ & $34,3(4,4)$ & $27,9(1,1)$ & $22,4(1,7)$ \\
\hline Diplôme postsecondaire ${ }^{\mathrm{a}}$ (en \%) & 55,5 & 59,9 & 60,8 \\
\hline $\begin{array}{l}\text { Revenu personnel total moyen } \\
\text { (en dollars) (ÉT) }^{\mathrm{a}}\end{array}$ & $41904,9(40 \quad 181,8)$ & 44 135,8 (43 876,6) & 37 176,5 (37 545,7) \\
\hline Citadins $^{\mathrm{a}}(\mathrm{en} \%)$ & 78,2 & 80,7 & 84,3 \\
\hline Naissance au Canada (en \%) & 81,8 & 75,5 & 73,7 \\
\hline Identité autochtone ${ }^{c}$ (en \%) & 4,2 & 2,9 & 2,7 \\
\hline Type caucasien ${ }^{\mathrm{a}}$ (en \%) & 90,0 & 85,2 & 80,5 \\
\hline N’a jamais fuméa (en \%) & 32,5 & 34,6 & 38,6 \\
\hline $\begin{array}{l}\text { Consommation de cinq portions de fruits } \\
\text { et légumes ou plus par jour }{ }^{\mathrm{a}} \text { (en \%) }\end{array}$ & 61,0 & 57,9 & 54,3 \\
\hline $\begin{array}{l}\text { Perception de son poids : excès } \\
\text { de poids }{ }^{\mathrm{a}} \text { (en } \% \text { ) }\end{array}$ & 86,6 & 53,3 & 14,4 \\
\hline $\begin{array}{l}\text { État de santé général perçu excellent } \\
\text { ou très bon }{ }^{\mathrm{a}}(\mathrm{en} \%)\end{array}$ & 45,1 & 59,9 & 65,6 \\
\hline $\begin{array}{l}\text { Nombre moyen de consultations médicales } \\
\text { au cours de la dernière année (ÉT) }{ }^{c}\end{array}$ & $4,6(7,5)$ & $3,7(6,9)$ & $3,5(6,1)$ \\
\hline
\end{tabular}

Abréviations : ESCC, Enquête sur la santé dans les collectivités canadiennes; ÉT, écart-type; IMC, indice de masse corporelle.

a Les sous-ensembles de catégories de classification de l'IMC varient grandement les uns par rapport aux autres au seuil de 0,05 lors de la comparaison par le test $z$ de paires de catégories. Les données ont été ajustées pour les comparaisons multiples à l'aide de la correction de Bonferroni.

b La catégorie «poids normal » varie grandement par rapport aux autres catégories au seuil de 0,05 lors de la comparaison par le test $z$ de paires de catégories. Les données ont été ajustées pour les comparaisons multiples à l'aide de la correction de Bonferroni.

c La catégorie « obésité » varie grandement par rapport aux autres catégories au seuil de 0,05 lors de la comparaison par le test z de paires de catégories. Les données ont été ajustées pour les comparaisons multiples à l'aide de la correction de Bonferroni.

faible. Le coût élevé était un obstacle beaucoup plus important $(p<0,001)$ chez les personnes ayant fait un CCS (6\%) que chez celles n'en ayant pas fait (4\%), mais cet obstacle n'était pas très fréquent. Les écarts entre les personnes ayant fait un CCS et celles n'en ayant pas fait étaient non significatifs pour la fréquence des obstacles liés au manque de volonté ou d'autodiscipline, à l'emploi du temps professionnel et à une incapacité ou à un problème de santé.

Les premiers modèles de régression logistique ont indiqué que l'IMC, la perception de son poids, la consommation de fruits et de légumes, le nombre de consultations médicales, le tabagisme et l'état de santé général perçu représentaient des variables importantes liées aux CCS. Un effet d'interaction important entre l'âge et le tabagisme a été observé pour tous les modèles. La capacité d'explication de la variance s'est révélée limitée pour tous les modèles. Le modèle lié à l'augmentation de la pratique de sports ou d'exercice a révélé un $R^{2}$ de Nagelkerke de 0,07 , ce qui indique une faible explication de la variance, avec des résultats au test de Hosmer-Lemeshow significatifs ( $p=0,001$ ), signe d'un faible ajustement du modèle. Le modèle lié à la perte de poids donnait un $R^{2}$ de Nagelkerke de 0,10 , mais un test de Hosmer-Lemeshow non significatif $(p=0,12)$, donc un bon ajustement aux données. Le modèle lié à l'amélioration des habitudes alimentaires donnait un $R^{2}$ de Nagelkerke de 0,06, et un test de Hosmer-Lemeshow non significatif $(p=0,09)$.

L'usage quotidien du tabac était inversement associé à l'augmentation de la pratique sportive ou d'exercice (rapport de cotes [RC] : 0,66; intervalle de confiance [IC] : 0,63 à 0,70 ), tandis que la perception de son poids et la consommation accrue de fruits et de légumes étaient associées positivement à l'augmentation de la pratique sportive ou d'exercice (voir le tableau 3). La perception de son poids, l'IMC et la consommation accrue de fruits et de légumes étaient positivement associés à la perte de poids, alors que le tabagisme et l'état de santé général perçu ne l'étaient pas. Enfin, l'usage quotidien du tabac était inversement associé à l'amélioration des habitudes alimentaires, mais la perception de son poids, l'IMC et la consommation accrue de fruits et de légumes y étaient positivement associés, aucune variable n'ayant été exclue du modèle.

\section{Analyse}

Le fait qu'environ $60 \%$ des adultes aient indiqué avoir fait un CCS au cours des 12 derniers mois est notable et laisse penser que de nombreux Canadiens sont à la fois préoccupés par leur santé et prêts à apporter des changements positifs à leur mode de vie. De même, que deux tiers des répondants aient ressenti le besoin d'en faire davantage pour améliorer leur santé suggère que cette préoccupation est généralisée. Comme il s'agissait d'un nouvel ensemble de questions à l'échelle nationale (elles avaient été incluses seulement dans un sous-ensemble de l'ESCC du cycle 3.1), il faudra attendre quelques années avant de recueillir des données comparables, que ce soit pour une comparaison longitudinale ou une comparaison transversale avec d'autres enquêtes.

Le fait d'insister sur l'identification du CCS le plus important était une nouveauté et a fait émerger de nouveaux enseignements par rapport aux études de population évaluant une gamme de $\operatorname{CCS}^{13,14}$. L'augmentation de la pratique sportive ou d'exercice a été mentionnée presque trois fois plus souvent que le deuxième CCS le plus fréquent, à savoir l'amélioration des habitudes alimentaires (29 \% contre $10 \%$ ). Ce résultat va à l'encontre d'autres études qui suggèrent que, chez les personnes en excès de poids, les changements liés à l'activité physique sont 
TABLEAU 2

Fréquence des caractéristiques de la population de 18 ans et plus relatives aux changements de comportement en matière de santé, par classe d'IMC, Canada, ESCC 2007

\begin{tabular}{|c|c|c|c|c|}
\hline \multirow[t]{2}{*}{ Variable } & \multicolumn{4}{|c|}{ Classification selon l'IMC } \\
\hline & $\begin{array}{c}\text { Obésité } \\
n=30442 \\
(\%)\end{array}$ & $\begin{array}{c}\text { Excès de poids } \\
n=31831 \\
(\%)\end{array}$ & $\begin{array}{c}\text { Poids normal } \\
\mathrm{n}=49176 \\
(\%)\end{array}$ & $\begin{array}{c}\text { Ensemble du } \\
\text { groupe } \\
\mathrm{n}=\mathbf{1 1 1} 449(\%)\end{array}$ \\
\hline \multicolumn{5}{|c|}{ A fait quelque chose pour améliorer sa santé } \\
\hline Oui $^{\mathrm{a}}$ & $18314(60,2)$ & $18607(58,5)$ & $27114(55,2)$ & $64035(57,5)$ \\
\hline Non $^{\mathrm{a}}$ & $12092(39,8)$ & $13190(41,5)$ & $22006(44,8)$ & $47288(42,5)$ \\
\hline Ne sait pas / Refus de répondre & 36 & 35 & 55 & 126 \\
\hline \multicolumn{5}{|l|}{ CCS le plus important } \\
\hline Sans objet ${ }^{\mathrm{a}}$ & $12092(39,8)$ & $13190(41,5)$ & $22007(44,9)$ & $47289(42,5)$ \\
\hline A fait plus d'exercice ou de sport ${ }^{b}$ & $7898(26,0)$ & $9460(29,8)$ & $14578(29,7)$ & $31936(28,7)$ \\
\hline A perdu du poids ${ }^{\mathrm{a}}$ & $3121(10,3)$ & $2315(7,3)$ & $1910(3,9)$ & $7346(6,6)$ \\
\hline A amélioré ses habitudes alimentaires ${ }^{\mathrm{a}}$ & $3156(10,4)$ & $3087(9,7)$ & $4502(9,2)$ & $10745(9,7)$ \\
\hline Autre $^{\mathrm{a}, \mathrm{c}}$ & $4113(13,5)$ & $3708(11,7)$ & $6069(12,3)$ & $13890(12,5)$ \\
\hline Ne sait pas / Refus de répondre & 25 & 37 & 55 & 117 \\
\hline Non précisé & 36 & 34 & 56 & 126 \\
\hline \multicolumn{5}{|l|}{ Pense qu'il y a autre chose à faire } \\
\hline Oui $^{\mathrm{a}}$ & $23405(77,5)$ & $21399(67,7)$ & $30913(63,2)$ & $75717(68,4)$ \\
\hline Non $^{\mathrm{a}}$ & $6801(22,5)$ & $10227(23,3)$ & $17989(36,8)$ & $35017(31,6)$ \\
\hline Ne sait pas / Refus de répondre & 201 & 172 & 219 & 589 \\
\hline Non précisé & 36 & 34 & 56 & 126 \\
\hline \multicolumn{5}{|c|}{ A fait face à des obstacles à l'amélioration de sa santé } \\
\hline Oui $^{\mathrm{a}}$ & $11885(50,9)$ & $10269(48,1)$ & $16039(52,0)$ & $38193(50,6)$ \\
\hline Non $^{\mathrm{a}}$ & $11452(49,1)$ & $11079(51,9)$ & $14790(48,0)$ & $37321(49,4)$ \\
\hline Sans objet & 6801 & 10227 & 17989 & 35017 \\
\hline Ne sait pas / Refus de répondre & 67 & 51 & 84 & 201 \\
\hline Non précisé & 236 & 206 & 274 & 717 \\
\hline
\end{tabular}

Abbréviations : CCS, changement de comportement en matière de santé; ESCC, Enquête sur la santé dans les collectivités canadiennes; IMC, indice de masse corporelle.

a Les sous-ensembles des catégories de classification de l'IMC varient grandement les uns par rapport aux autres au seuil de 0,05 lors de la comparaison par le test $z$ de paires de catégories. Les données ont été ajustées pour les comparaisons multiples à l'aide de la correction de Bonferroni.

b La catégorie « obésité » varie grandement par rapport aux autres catégories au seuil de 0,05 lors de la comparaison par le test $z$ de paires de catégories. Les données ont été ajustées pour les comparaisons multiples à l'aide de la correction de Bonferroni.

c Autres changements : a cessé de fumer ou a réduit sa consommation de tabac, a consommé moins d'alcool, a réduit son niveau de stress, a reçu des traitements médicaux, a pris des vitamines ou a effectué un autre changement non spécifié.

plus difficiles à réaliser que les changements liés à l'alimentation ${ }^{15}$.

Les écarts entre les études sont considérables. Newson et collab. ${ }^{16}$ ont étudié les CCS apportés après un diagnostic de maladie chronique au sein de la cohorte de l'Enquête nationale sur la santé de la population. Parmi les personnes s'étant mises à souffrir de diabète, et à qui les médecins recommandent généralement de faire plus d'exercice et d'améliorer leurs habitudes alimentaires ${ }^{17}$, le pourcentage de personnes actives physiquement et consommant cinq portions ou plus de fruits et légumes par jour a augmenté de $7 \%$, seulement 35 des 487 personnes interrogées ayant adopté ces deux comportements. Chez les personnes atteintes d'une cardiopathie, le pourcentage de personnes respectant les lignes directrices en matière d'activité physique n'a augmenté que de $2 \%$ (de 51,6 \% à 53,9\%), mais on a assisté à une hausse de $9 \%$ (de $42 \%$ à $51 \%$ ) de la consommation recommandée de fruits et légumes. Les raisons de ces écarts demeurent incertaines, mais il semble que les sujets plus âgés soient moins enclins à changer leurs habitudes ${ }^{16}$.

L'examen des résultats de la régression logistique exploratoire de notre étude suggère lui aussi des écarts dans les comportements en matière de santé en fonction de sous-groupes de population. Diverses formes d'analyses en grappe permettraient de mieux caractériser les groupes de population les plus susceptibles d'apporter des changements à leur mode de vie. Dans l'ensemble, la proportion élevée de personnes ayant déclaré avoir augmenté la fréquence de ses activités physiques était encourageante.

Un des objectifs de notre étude consistait à évaluer la fréquence globale des principaux CCS en lien avec la prise en charge de son poids (régime alimentaire, exercice, perte de poids) par le biais d'une comparaison entre groupes de répondants classés par catégorie d'IMC (catégories préalablement définies en fonction du risque de mortalité connexe, soit poids normal, excès de poids et obésité). Il convient de souligner que la perte de poids en tant que CCS principal était relativement rare (10\% des répondants obèses), ce qui conforte les nombreuses données probantes prouvant que la perte de poids constitue un défi. Obtenir des données en population de cette nature est important, car la majorité des tentatives de perte de poids se produisent en marge du système de soins de santé et ne font l'objet d'aucun suivi. Les répondants à l'ESCC n’ont pas été invités à fournir de détails à ce sujet mais, dans une étude reposant sur des données de la National Health and Nutrition Examination Survey (NHANES) 1999-2004 limitée aux répondants atteints d'obésité abdominale et de maladies cardiovasculaires, $15 \%$ ont déclaré avoir perdu $5 \%$ ou plus de leur poids ${ }^{18}$. Les répondants obèses ayant participé à une entrevue en personne pour la NHANES 2001-2006 ont déclaré en proportion beaucoup plus élevée (38\%) avoir perdu $5 \%$ ou plus de leur poids ${ }^{19}$. Dans le même ordre d'idée, $35 \%$ des adultes obèses ayant participé au programme de perte de poids Counterweight dans le cadre de soins primaires ont perdu $5 \%$ ou plus 
FIGURE 2

Comparaison des motifs à l'origine des obstacles aux changements comportementaux en matière de santé par catégorie d'IMC

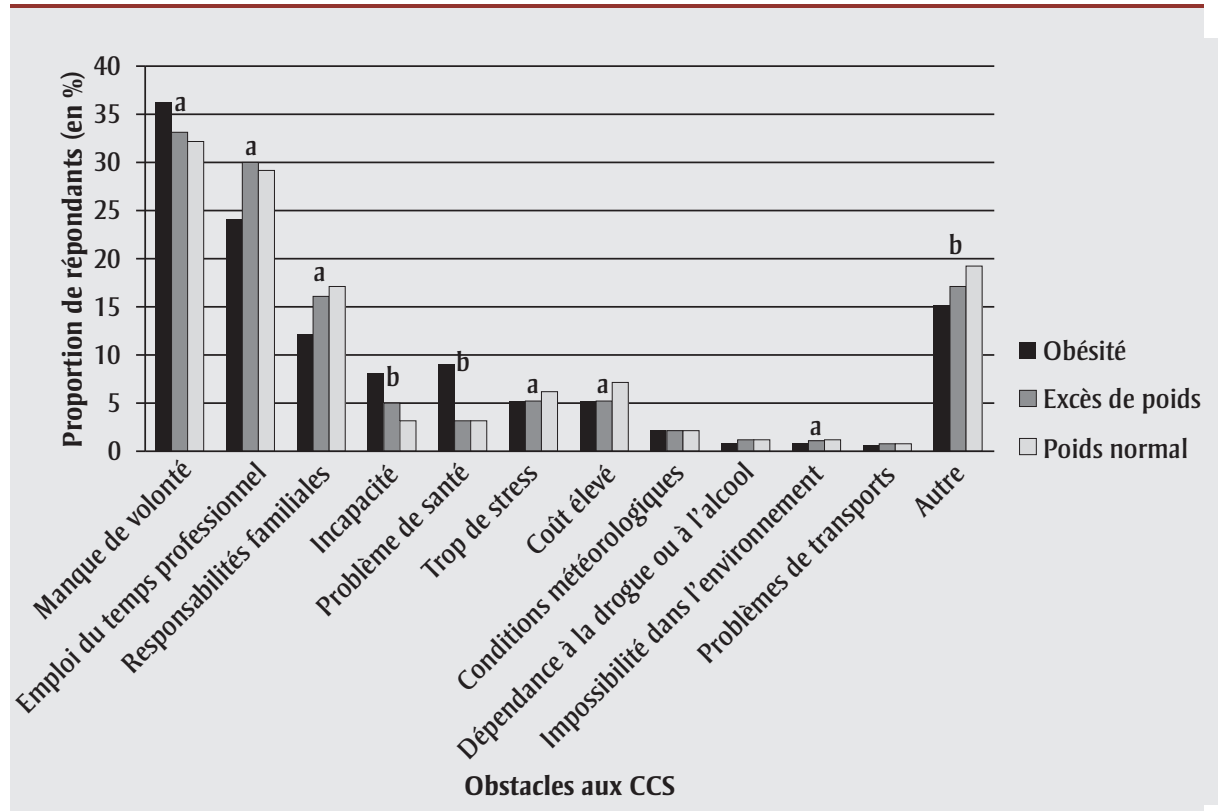

Abréviations : CCS, changement de comportement en matière de santé; IMC, indice de masse corporelle.

${ }^{a}$ Les groupes d'IMC des personnes dont le poids est normal et des personnes obèses varient grandement à $p<0,05$.

${ }^{\mathrm{b}}$ Tous les groupes d'IMC varient grandement à $p<0,05$.

de leur poids ${ }^{20}$, mais la moitié d'entre eux avait abandonné le programme avant la fin de la période de suivi d'un an. Des enquêtes de population plus détaillées sont nécessaires pour déterminer le pourcentage de Canadiens capables de présenter une perte de poids cliniquement valable et pour définir les caractéristiques de ces personnes.

Comparativement aux personnes dont le poids était normal, la majorité des personnes en excès de poids et obèses ont apporté des CCS de tous types et ont affirmé vouloir en faire davantage pour améliorer leur santé. À l'instar des répondants dont le poids était normal, ils ont également déclaré que l'exercice représentait leur CCS le plus important. Compte tenu des effets positifs pour tous de l'exercice sur la santé, quel que soit le poids, ces résultats suggèrent que le fait de valoriser l'exercice dans le cadre d'efforts de promotion de la santé serait bien accueilli. À l'heure actuelle, les programmes de promotion de la santé prônant l'atteinte d'un "poids santé » peuvent être très décourageants pour les personnes en excès de poids ou obèses, étant donné la difficulté à perdre du poids. Un message positif fondé sur l'exercice correspondrait mieux aux préférences de la population et serait plus conforme aux nouvelles données probantes qui indiquent que les personnes obèses en bonne santé physique et métabolique présentaient un risque de mortalité par maladie cardiovasculaire moins élevé que celles ne l'étant pas ${ }^{21,22}$.

L'évaluation de la fréquence des obstacles est une spécificité de l'ESCC : nous n'avons trouvé aucune autre enquête d'importance évaluant ce type d'éléments. La moitié des personnes répondant aux critères de l'étude ont affirmé ne pas avoir rencontré d'obstacles à leurs CCS. Le manque de volonté s'est révélé, comme prévu, l'obstacle le plus fréquemment cité, suivi par l'emploi du temps professionnel et les responsabilités familiales.

La " volonté » renvoie à deux concepts, l'auto-efficacité et la contrôlabilité, qui sont les thèmes majeurs de la plupart de la littérature sur les comportements en matière de santé, ainsi que l'ont montré des textes fondateurs comme Health Behavior and Health Education, publiés en
1990 , et qui ont fourni le premier aperçu d'ensemble des principales théories de l'époque ${ }^{23}$. Depuis, l'importance du rôle joué par les contextes environnementaux et sociaux sur le comportement a été largement reconnue. Le manque de temps, qui est l'un des obstacles aux CCS le plus souvent cité dans la littérature ${ }^{24,25,26}$, ne figure pas dans l'ESCC comme obstacle en tant que tel. Toutefois, comme le travail et les responsabilités familiales sont des tâches intenses qui réduisent le temps disponible pour apporter des CCS, on peut considérer que ces tâches sont des obstacles assimilables au manque de temps. Si l'on analyse les obstacles en fonction de l'IMC, on remarque que les répondants obèses ont rapporté une fréquence d'obstacles similaire à celle des répondants dont le poids était normal (51\% par rapport à $52 \%$ ), mais que les types d'obstacles différaient quelque peu, l'incapacité et le manque de volonté étant plus fréquents chez les personnes obèses et le travail et les responsabilités familiales moins fréquents. Dans l'ensemble, les transports, le coût élevé, le stress et le manque de disponibilité ne constituaient pas des obstacles fréquents pour la majorité des Canadiens. Ce type de données en population sur la fréquence des principaux obstacles aux CCS pourraient se révéler important pour la conception de nouvelles approches.

Notre modèle de régression logistique était de nature exploratoire et avait comme objectif de repérer les corrélations possibles au-delà des écarts manifestes liés à l'âge, au sexe, au revenu et au niveau de scolarité. L'association entre l'IMC et les CCS a déjà été étudiée : Verheijden et collab $^{27}$. ont observé que les répondants obèses étaient plus susceptibles de continuer à participer à un programme de promotion des CCS que les répondants dont le poids était normal, tandis que Teixeria et collab ${ }^{28}$. ont relevé une corrélation positive entre l'IMC et la perte de poids.

Le tabagisme a souvent été inversement associé aux $\operatorname{CCS}^{27,29,30}$. La consommation accrue de fruits et légumes ayant été associée à une meilleure santé dans de nombreuses études épidémiologiques, il n'est pas étonnant que les personnes qui mangent déjà beaucoup de fruits et de 
TABLEAU 3

Résumé des modèle de régression des changements de comportement en matière de santé, population de 18 ans et plus, Canada, ESCC 2007

\begin{tabular}{|c|c|c|c|c|c|c|}
\hline & \multicolumn{2}{|c|}{$\begin{array}{l}\text { Auǵmentation de la } \\
\text { pratique de sports } \\
\text { ou d'exercice }\end{array}$} & \multicolumn{2}{|c|}{ Perte de poids } & \multicolumn{2}{|c|}{$\begin{array}{c}\text { Amélioration } \\
\text { des habitudes } \\
\text { alimentaires }\end{array}$} \\
\hline & RC & IC à $95 \%$ & RC & IC à $95 \%$ & $\mathbf{R C}$ & IC à 95 \% \\
\hline IMC (par unité d'augómentation) & - & - & 1,06 & 1,05 à 1,07 & 1,02 & 1,01 à 1,02 \\
\hline \multicolumn{7}{|l|}{ Tabagisme } \\
\hline N’a jamais fuméa & - & - & - & - & - & - \\
\hline Fumeur quotidien & 0,66 & 0,63 à 0,70 & - & - & 0,72 & 0,66 à 0,77 \\
\hline $\begin{array}{l}\text { Fumeur occasionnel anciennement } \\
\text { quotidien }\end{array}$ & 1,04 & 0,94 à 1,16 & - & - & 0,99 & 0,85 à 1,16 \\
\hline Fumeur occasionel & 1,18 & 1,04 à 1,34 & - & - & 0,88 & 0,72 à 1,08 \\
\hline Ex-fumeur quotidien & 1,13 & 1,08 à 1,19 & - & - & 1,14 & 1,06 à 1,22 \\
\hline Ex-fumeur occasionnel & 1,08 & 1,02 à 1,14 & - & - & 1,22 & 1,13 à 1,32 \\
\hline \multicolumn{7}{|l|}{ Perception de son poids ${ }^{b}$} \\
\hline À peu près correct ${ }^{\mathrm{a}}$ & - & - & - & - & - & - \\
\hline Excès de poids & 1,27 & 1,23 à 1,32 & 1,79 & 1,66 à 1,94 & 1,26 & 1,18 à 1,34 \\
\hline Poids insuffisant & 1,04 & 0,94 à 1,15 & 0,93 & 0,73 à 1,19 & 0,90 & 0,77 à 1,06 \\
\hline \multicolumn{7}{|l|}{ Consommation de fruits et légumes ${ }^{c}$} \\
\hline Moins de 5 portions par jour ${ }^{\mathrm{a}}$ & - & - & - & - & - & - \\
\hline 5 à 10 portions par jour & 1,28 & 1,23 à 1,33 & 1,42 & 1,33 à 1,52 & 1,45 & 1,37 à 1,53 \\
\hline Plus de 10 portions par jour & 1,33 & 1,22 à 1,45 & 1,55 & 1,34 à 1,81 & 1,86 & 1,65 à 2,08 \\
\hline Nombre de consultations médicales ${ }^{\mathrm{d}}$ & 1,01 & 1,01 à 1,01 & 1,02 & 1,02 à 1,02 & 1,01 & 1,01 à 1,02 \\
\hline \multicolumn{7}{|l|}{ État de santé général perçu ${ }^{\mathrm{e}}$} \\
\hline Excellent $^{\mathrm{a}}$ & - & - & - & - & - & - \\
\hline Mauvais/médiocre & 1,01 & 0,94 à 1,09 & - & - & 1,52 & 1,36 à 1,69 \\
\hline Bon & 1,12 & 1,06 à 1,18 & - & - & 1,45 & 1,34 à 1,57 \\
\hline Très bon & 1,17 & 1,12 à 1,23 & - & - & 1,37 & 1,27 à 1,47 \\
\hline
\end{tabular}

Abréviations : CCS, changement de comportement en matière de santé; ESCC, Enquête sur la santé dans les collectivités canadiennes; IC, intervalle de confiance; IMC, indice de masse corporelle; RC, rapport de cotes.

a Catégorie de référence.

b Cette variable sert à classer le répondant en fonction de la perception qu'il a de son poids.

c Cette variable sert à classer le répondant en fonction du nombre de portions de fruits et légumes consommées chaque jour, d'après un rappel des aliments consommés dans une journée.

${ }^{\text {d }}$ Cette variable fournit le nombre de rencontres ou de discussions avec un médecin de famille ou un médecin spécialiste au cours des 12 derniers mois.

e Cette variable fournit l'état de santé du répondant selon son jugement.

légumes soient plus enclines à faire des CCS. La perception d'un excès de poids et d'un mauvais état de santé général, ainsi que le nombre élevé de consultations médicales sont des facteurs associés aux CCS, comme cela a également été démontré dans des études antérieures ${ }^{31}$. Une meilleure caractérisation des sous-groupes qui entreprennent des CCS est nécessaire pour orienter les efforts de promotion de la santé. Il faudrait analyser chacune des variables de façon plus approfondie, car il pourrait s'agir d'indicateurs indirects de mode de vie ou d'autres facteurs pouvant à leur tour être associés aux CCS. D’autres recherches sont donc nécessaires pour comprendre pleinement ces relations.

Seule une autre étude ${ }^{32}$ a porté sur les CCS mesurés par l'ESCC cycle 4.1, et les résultats de Hystad et Carpiano quant à la fréquence globale des CCS ont confirmé les nôtres. Leur travail était axé sur le sentiment d'appartenance à la collectivité.
Dans notre analyse, le sentiment d'appartenance a été inclus en tant que prédicteur potentiel dans les modèles de régression initiaux mais, bien qu'il soit statistiquement significatif, il a été largement éclipsé par d'autres variables et comptait à peine dans la variance du modèle. Par conséquent, par souci de concision, nous l'avons retiré du modèle final.

\section{Forces et limites}

Cette étude a permis d'estimer la fréquence des CCS les plus importants et de caractériser les obstacles aux changements par catégorie d'IMC au sein de la population canadienne. Il sera possible, de ce fait, de mieux évaluer au fil du temps les résultats des efforts de promotion de la santé à l'échelle de la population.

Notre étude comporte aussi des limites notables. Certes, les mesures relatives au CCS comprises dans l'ESCC cycle 4.1 ont apporté de nouveaux enseignements importants, mais nous n'avons pas été en mesure de trouver, dans la documentation de Statistique Canada ou dans la littérature évaluée par les pairs, d'évaluations publiées portant sur la fiabilité ou la validité des différents types de questions. Il a été mentionné que l'avis d'experts avait été sollicité au sujet des mesures ${ }^{12}$, ce qui fait que les mesures actuelles constituent un point de départ. Il faut toutefois valider le concept selon lequel la perte de poids, l'exercice et le changement de régime alimentaire représentent des CCS distincts dans les enquêtes en population, étant donné qu'il faut généralement apporter des changements au régime alimentaire et à la pratique de l'activité physique pour perdre du poids. Il est urgent d'effectuer ce type de travaux, car la capacité de surveillance des CCS dans la population pourrait se révéler un ajout précieux aux outils déjà utilisés pour évaluer les CCS. De plus, on ne connaît pas les avantages relatifs de rendre compte, comme c'est le cas dans notre étude, des CCS les plus importants plutôt que des divers CCS en même temps. De même, il est nécessaire de mener d'autres études pour évaluer la fiabilité et la validité de l'autodéclaration des obstacles aux changements. 
La définition de l'obésité en fonction de l'IMC constitue une autre limite de cette étude et d'autres enquêtes sur la santé de la population, car il est de plus en plus évident que l'IMC à lui seul ne peut être un indicateur satisfaisant de santé ou de risque de mortalité. Il faudra faire appel à diverses stratégies pour régler cette question, notamment adopter des approches axées davantage sur les sous-échantillons ${ }^{19,20}$.

\section{Conclusion}

Notre analyse des CCS autodéclarés et des obstacles aux CCS dans l'ESCC cycle 4.1 a révélé une fréquence globale élevée des CCS, en particulir de l'augmentation de l'exercice, et ce, par une minorité notable d'adultes. Nous étions tout spécialement intéressés par les écarts possibles en fonction de l'obésité, mais notre analyse a révélé que les CCS autodéclarés des personnes obèses et des personnes dont le poids était normal étaient très similaires. Les analyses de régression ont permis de cerner d'autres facteurs susceptibles d'aider à une meilleure caractérisation de la population. Même si les méthodes d'évaluation des CCS et des obstacles qui leur sont liés au sein de la population doivent être améliorées, notre étude a fourni des renseignements utiles pour l'élaboration de nouvelles stratégies en matière de CCS.

\section{Références}

1. Conseil canadien de la santé. Changement de vitesse : cibler, non plus les soins de santé au Canada, mais un Canada en santé. Toronto (Ont.) : Conseil canadien de la santé; 2010

2. Statistique Canada. Enquête canadienne sur les mesures de la santé : tableaux de données du cycle 2, 2009 à 2011. Ottawa (Ont.) : Gouvernement du Canada; 2012.

3. Statistique Canada. Tableaux sommaires Tableaux par sujet [Internet]. Ottawa (Ont.) : Gouvernement du Canada; [consultation le 3 octobre 2013]. Consultable en ligne à la page : http://www.statcan.gc.ca/tables-tableaux /sum-som/102/ind01/13_2966_2968-fra.htm? hili_none
4. Plotnikoff RC, Lippke S, Johnson ST, Hugo K, Rodgers W, Spence JC. Awareness of Canada's Physical Activity Guide to Healthy Active Living in a large community sample. Am J Health Promot. 2011;25:294-7.

5. Garcia AC, Piche LA. Perceptions and use of Canada's Food Guide to Healthy Eating by grocery shoppers in London, Ontario. Can J Diet Pract Res. 2001;62:123-127.

6. Statistique Canada. Enquête sur la santé dans les collectivités canadiennes Composante annuelle (ESCC), Information détaillée pour 2007 (Cycle 4.1) [Internet]. Ottawa (Ont.) : Statistique Canada; [modifié le 17 juin 2008; consultation le 3 octobre 2013]. Consultable en ligne à la page : http://www23.statcan.gc.ca/imdb/p2SV_f .pl? Function $=$ getSurvey\&SurvId $=50653 \&$ InstaId $=29539 \&$ SDDS $=3226$

7. Santé Canada. Lignes directrices pour la classification du poids chez les adultes. Ottawa (Ont.) : Santé Canada; 2003.

8. Gorber SC, Tremblay MS. The bias in selfreported obesity from 1976 to 2005 : a Canada-US comparison. Obesity. 2010;18(2): 354-361.

9. Gorber SC, Shields M, Tremblay MS, McDowell I. La possibilité d'établir des facteurs de correction applicables aux estimations autodéclarées de l'obésité. Rapports sur la santé. 2008;19(3):74-87.

10. Ajzen I. Perceived behavioral control, selfefficacy, locus of control, and the theory of planned behavior. J Appl Social Psychol. 2002;32(4):665-83.

11. Strecher VJ, DeVellis BM, Becker MH, Rosenstock IM. The role of self-efficacy in achieving health behavior change. Health Educ Q. 1986;13(1):73-92.

12. Statistique Canada. Enquête sur la santé dans les collectivités canadiennes (ESCC) Fichiers de microdonnées 2007 - Guide de l'utilisateur. Ottawa (Ont.) : Statistique Canada; 2008. PDF (575 Ko) téléchargeable à partir du lien : http://www23.statcan.gc. ca/imdb-bmdi/document/3226_D7_T9_V4fra.pdf

13. Prochaska JJ, Spring B, Nigg CR. Multiple health behavior change research: an introduction and overview. Prev Med. 2008; 46(3):181-188.
14. Lippke S, Nigg CR, Maddock JE. Healthpromoting and health-risk behaviors : theory-driven analyses of multiple health behavior change in three international samples. Int J Behav Med. 2012;19(1):1-13.

15. Bish CL, Blanck HM, Maynard LM, Serdula MK, Thompson NJ, Khan LK. Activity/ participation limitation and weight loss among overweight and obese US adults : 1999 to 2002 NHANES. MedGenMed. 2007; $9(3): 63$.

16. Newson JT, Huguet N, Ramage-Morin PL et collab. Modifications des comportements influant sur la santé après le diagnostic d'une maladie chronique chez les Canadiens de 50 ans et plus. Rapports sur la santé. 2012;23(4):1-8.

17. Comité d'experts des Lignes directrices de pratique clinique de l'Association canadienne du diabète. Lignes directrices de pratique clinique 2013 de l'Association canadienne du diabète pour la prévention et le traitement du diabète au Canada. Can J Diabetes. 2013;37(suppl 5):S361-S598.

18. Singh S, Somers VK, Clark MM et collab. Physician diagnosis of overweight status predicts attempted and successful weight loss in patients with cardiovascular disease and central obesity. Am Heart J. 2010;160(5): 934-942.

19. Nicklas JM, Huskey KW, Davis RB, Wee CC. Successful weight loss among obese U.S. adults. Am J Prev Med. 2012;42(5):481-485.

20. Counterweight Project Team. The implementation of the Counterweight Programme in Scotland, UK. Fam Pract. 2012;29(Suppl 1): i139-144.

21. Hamer M, Stamatakis E. Metabolically healthy obesity and risk of all-cause and cardiovascular disease mortality. J Clin Endocrinol Metab. 2012;97(7):2482-2498.

22. Ortega FB, Lee DC, Katzmarzyk PT, et collab. The intriguing metabolically healthy but obese phenotype : cardiovascular prognosis and role of fitness. Eur Heart J. 2013;34(5):389-397.

23. Glanz K, Lewis FM, Rimer BK (dir.). Health behavior and health education: theory, research and practice. San Francisco (CA) : Jossey-Bass; 1990. 
24. Ruelaz AR, Diefenbach P, Simon B, Lanto A, Arterburn D, Shekelle PG. Perceived barriers to weight management in primary care--perspectives of patients and providers. J Gen Intern Med. 2007;22(4):518522.

25. Mauro M, Taylor V, Wharton S, Sharma AM. Barriers to obesity treatment. Eur J Intern Med. 2008;19(3):173-180.

26. Welch N, McNaughton SA, Hunter W, Hume C, Crawford D. Is the perception of time pressure a barrier to healthy eating and physical activity among women? Public Health Nutr. 2009;12(7):888-895.

27. Verheijden MW, Jans MP, Hildebrandt VH, Hopman-Rock M. Rates and determinants of repeated participation in a web-based behavior change program for healthy body weight and healthy lifestyle. J Med Internet Res. 2007;9(1):e1.

28. Teixeria PJ, Going SB, Sardinha LB, Lohman TG. A review of psychosocial pre-treatment predictors of weight control. Obesity Rev. 2005;6(1):43-65.

29. Van Horn L, Dolecek TA, Grandits GA, Skweres L. Adherence to dietary recommendations in the special intervention group in the Multiple Risk Factor Intervention Trial. Am J Clin Nutr. 1997;65(1 Suppl):289S$304 S$.

30. Stubbs J, Whybrow S, Teixeira P, et collab. Problems in identifying predictors and correlates of weight loss and maintenance: implications for weight control therapies based on behaviour change. Obes Rev. 2011;12(9):688-708.

31. Yaemsiri S, Slining MM, Agarwal SK. Perceived weight status, overweight diagnosis, and weight control among US adults: The NHANES 2003-2008 study. Int J Obes. 2011;35(8):1063-1070.

32. Hystad P, Carpiano RM. Sense of community-belonging and health-behaviour change in Canada. J Epidemiol Community Health. 2012;66(3):277-283. 